\title{
Investigação da incidência de cefaleia pós-punção dural em um hospital na cidade de
} Três Lagoas/MS

\author{
Investigation of the incidence of post-puncture headache in a hospital in the city of Três Lagoas/MS \\ Investigación de la incidencia de cefalea pospunción en un hospital de la ciudad de Três Lagoas/MS
}

Recebido: 09/12/2021 | Revisado: 17/12/2021 | Aceito: 22/12/2021 | Publicado: 04/01/2022

\author{
Ana Paula Paschoal \\ ORCID: https://orcid.org/0000-0003-0729-2723 \\ Universidade Federal de Mato Grosso do Sul, Brasil \\ E-mail: appaschoal3@gmail.com \\ Lara Cristina Rodrigues de Oliveira Costa \\ ORCID: https://orcid.org/0000-0003-4153-2225 \\ Universidade Federal de Mato Grosso do Sul, Brasil \\ E-mail: lara_cristina2408@hotmail.com \\ Marcello Pansani Vilaça \\ ORCID: https://orcid.org/0000-0002-5823-0971 \\ Universidade Federal de Mato Grosso do Sul, Brasil \\ E-mail: marcellopansani@gmail.com \\ Kelly Regina Torres-da-Silva \\ ORCID: https://orcid.org/0000-0003-4108-3191 \\ Faculdades Integradas de Três Lagoas, Brasil \\ E-mail: kellytorresdasilva1@gmail.com \\ André Valério Silva \\ ORCID: https://orcid.org/0000-0003-0309-5394 \\ Universidade Federal de Mato Grosso do Sul, Brasil \\ E-mail: andre.valerio@ufms.br
}

\begin{abstract}
Resumo
A cefaleia pós-punção dural (CPPD) é a complicação mais frequente da anestesia subaracnóidea, com incidência variando entre 0,04 a 3\%. Surge, geralmente, em até sete dias após a punção dural, com duração aproximada de duas semanas e, normalmente, é autorresolutiva. Durante o período da dor, o paciente pode ter sua rotina diária prejudicada, pois o quadro tipicamente piora quando é adotada a posição ortostática. Diversos fatores de risco podem contribuir para o aparecimento da complicação, e eles podem ser relacionados ao paciente, à técnica de punção e ao material utilizado. O diagnóstico de CPPD é clínico, e a conduta terapêutica varia de acordo com a apresentação e gravidade da dor, podendo ser clínica ou, idealmente, ser realizado o tamponamento sanguíneo peridural ou "blood patch". Objetivou-se identificar a frequência da CPPD tratada com tamponamento sanguíneo peridural, associada a fatores clínicos e sociodemográficos em um hospital na cidade de Três Lagoas/MS. Trata-se de estudo descritivo, tipo coorte transversal retrospectivo, via análise documental para a investigação de tamponamento sanguíneo peridural realizado a partir de cefaleia secundária, em pacientes que foram submetidos à anestesia subaracnóidea, no período de 01/01/2011 a 03/04/2019. Foram identificados 57 tamponamentos sanguíneos peridurais, com uma taxa de prevalência de $0,25 \%$, incidência anual oscilante e predomínio do sexo feminino. Do total, 49 (86\%) pacientes eram mulheres, $36(63 \%)$ tinham entre 21 e 40 anos e $52(91 \%)$ deles apresentaram os sintomas da CPPD em até 5 dias pósanestesia. Concluiu-se que a realização de tamponamento sanguíneo peridural para tratamento de CPPD ainda é comum e sem resolução definitiva. A necessidade da adoção constante de medidas profiláticas evidentes que possam reduzir a incidência da complicação é absoluta, uma vez que o retorno do paciente ao centro cirúrgico é acompanhado de riscos infectopatológicos, psicossociais e impactos socioeconômicos.
\end{abstract}

Palavras-chave: Cefaleia; Raquianestesia; Tamponamento interno; Incidência.

\begin{abstract}
Post-dural puncture headache (PDH) is the most frequent complication of subarachnoid anesthesia, with an incidence ranging from 0.04 to $3 \%$. It usually appears within seven days after a dural puncture, lasting approximately two weeks and is usually self-healing. During the period of pain, the patient may have his daily routine impaired, as the condition typically worsens when the orthostatic position is adopted. Several risk factors can contribute to the appearance of the complication, and they can be related to the patient, the puncture technique and the material used. The diagnosis of $\mathrm{PDH}$ is clinical, and the therapeutic approach varies according to the presentation and severity of the pain, and may be clinical or, ideally, epidural blood patch. The objective of this study was to identify the frequency of PDH treated with
\end{abstract}


blood patch, associated with clinical and sociodemographic factors in a hospital in the city of Três Lagoas/MS. This is a descriptive, retrospective cross-sectional cohort study, via document analysis for the investigation of epidural blood patch performed from secondary headache, in patients who underwent subarachnoid anesthesia, from 01/01/2011 to 04/03/2019. 57 epidural blood buffers were identified, with a prevalence rate of $0.25 \%$, annual fluctuating incidence and a predominance of females. Of the total, 49 (86\%) were women, $36(63 \%)$ patients were between 21 and 40 years old and $52(91 \%)$ of them had PDH symptoms within 5 days post-anesthesia. It can be concluded that epidural blood packing for the treatment of PDH is still common and without definitive resolution. The need for constant adoption of evident prophylactic measures that can reduce the incidence of the complication is absolute, since the patient's return to the operating room is accompanied by infectious, psychosocial and socioeconomic impacts.

Keywords: Headache; Spinal anesthesia; Blood patch; Incidence.

\section{Resumen}

La cefalea pospunción dural (CPPD) es la complicación más frecuente de la anestesia raquídea, con una incidencia que oscila entre el 0,04 y el 3\%. Por lo general, aparece dentro de los siete días posteriores a la punción dural, dura aproximadamente dos semanas y generalmente se resuelve por sí solo. Durante el período de dolor, el paciente puede tener alterada su rutina diaria, ya que la condición generalmente empeora cuando se adopta la posición de pie. Varios factores de riesgo pueden contribuir a la aparición de la complicación, y pueden estar relacionados con el paciente, la técnica de punción y el material utilizado. El diagnóstico de CPPD es clínico y el abordaje terapéutico varía según la presentación y la gravedad del dolor, puede ser clínico o, idealmente, se puede realizar un taponamiento sanguíneo epidural o "parche de sangre". El objetivo fue identificar la frecuencia de CPPD tratada con taponamiento sanguíneo epidural, asociada a factores clínicos y sociodemográficos en un hospital de la ciudad de Três Lagoas / MS. Se trata de un estudio de cohorte descriptivo, retrospectivo, transversal, mediante análisis documental para la investigación del taponamiento sanguíneo epidural por cefalea secundaria, en pacientes sometidos a raquianestesia, desde el 01/01/2011 hasta el 03/04 / 2019. Se identificaron un total de 57 parches sanguíneos epidurales, con una tasa de prevalencia del 0,25\%, incidencia anual oscilante y predominio del sexo femenino. Del total, 49 (86\%) eran mujeres, 36 (63\%) pacientes tenían entre 21 y 40 años y $52(91 \%)$ de ellos tenían síntomas de CPPD en los 5 días posteriores a la anestesia. Se concluyó que la realización del taponamiento sanguíneo epidural para el tratamiento de la CPPD sigue siendo común y no tiene una resolución definitiva. La necesidad de una constante adopción de medidas profilácticas evidentes que puedan reducir la incidencia de complicaciones es absoluta, ya que el regreso del paciente al quirófano se acompaña de riesgos infecciosos, psicosociales e impactos socioeconómicos.

Palabras clave: Dolor de cabeza; Anestesia espinal; Almacenamiento en búfer interno; Incidencia.

\section{Introdução}

A anestesia do neuroeixo, que inclui técnicas subdurais, epidurais, e a combinação destas duas, permite a realização de procedimentos cirúrgicos e outras intervenções dolorosas de maneira rápida e segura, produzindo analgesia, redução da ansiedade (ou ausência de consciência, quando acompanhada de anestesia geral) e relaxamento muscular adequado e profícuo (Falk et al., 2018; Ituk \& Wong, 2018).

A anestesia subaracnoidea, também conhecida como raquianestesia, se baseia na introdução de anestésico local no espaço subaracnóideo da região espinal, com o objetivo de bloquear temporariamente a transmissão de potenciais de ação tanto dentro da medula espinal quanto nas raízes dos nervos periféricos (Imbelloni, 2013).

As complicações decorrentes de anestesia subaracnóidea são raras (risco inferior a 0,04\%), uma vez que a técnica foi sendo acurada de forma a possibilitar elevado grau de segurança. Dentre as situações e grupos de risco que podem desencadear complicações neurológicas, os mais frequentes são: injeção intraneural de anestésico local, contaminação de seringas com detergentes e antissépticos, infecção por microrganismos, oxidantes presentes na preparação comercial de anestésicos locais, elevadas concentrações de anestésico local sobre o tecido nervoso, pacientes obesos e diabéticos. A partir da ocorrência de uma complicação, o diagnóstico e tratamento precoce podem evitar lesões irreversíveis e alterar o prognóstico do paciente (Ganem et al., 2002; Imbelloni, 2013).

Das possíveis complicações decorrentes da anestesia subaracnóidea, a cefaleia pós-punção dural (CPPD) é a mais frequente, e a incidência varia entre 0,04 a 3\%. O tipo de cefaleia que caracteriza a CPPD é a decorrente de hipotensão do LCS, que ocorre devido a extravasamento de LCS durante o procedimento anestésico, decorrente da permanência da vazão de 
LCS através do orifício produzido na dura-máter para realização da anestesia subaracnóidea. O orifício na dura-máter pode ter essa regeneração mais lenta devido à natureza não coagulável do LCS, portanto a trama tecidual que compõe a meninge deve se reorganizar de maneira a vedar o local da punção. Alguns tipos de CPPD são tão graves que chegam a ser incapacitantes, no entanto, o tempo médio de duração da CPPD é de cinco dias, e a maioria dos pacientes se recupera espontaneamente em sete dias (Netto et al, 2010; Drake, 2015).

Quando o paciente está em decúbito dorsal, a pressão do LCS na região lombar varia de 5-15 cm H2O, e aumenta para aproximadamente $40 \mathrm{~cm} \mathrm{H} 2 \mathrm{O}$ quando o paciente fica na posição ereta. A CPPD aparece quando o paciente eleva a cabeça em relação ao tórax, que acontece quando senta ou fica em pé, e isso ocorre porque, numa condição de baixo volume liquórico, o LCS migra para o espaço subaracnóideo vertebral pela ação da gravidade, aumenta a pressão transdural, e extravasa através do orifício de perfuração. As estruturas intracranianas (meninges, vasos e nervos), por suas vezes, sofrem tração devido à diminuição da pressão do LCS no espaço subaracnóideo encefálico, e isso pode explicar, pelo menos em parte, o componente doloroso da CPPD. Dependendo da estrutura craniana estimulada pela tração, a dor pode ser referida na região frontal (estímulo dos ramos do V nervo craniano - nervo trigêmeo), occipital (ramos do IX e X nervos cranianos - nervos glossofaríngeo e vago), pescoço e ombros (ramos dos nervos cervicais $\mathrm{C} 1, \mathrm{C} 2$ e C3), porém outros pacientes podem referir dor difusa (Wu et al., 2004; Imbelloni, 2013).

O componente doloroso da CPPD também pode ter contribuição da dilatação vascular venosa intracraniana, que acontece como forma de compensação da diminuição da pressão do LCS. Esse evento é explicado pela teoria de Monro-Kellie, a qual sugere que a soma do volume do conteúdo intracraniano é sempre constante, e os componentes que a compõem são: volume do parênquima encefálico (que é inelástico), volume do LCS e volume sanguíneo intracraniano. Dessa forma, se um componente da soma alterar sua contribuição para o volume total imutável, outro componente deve ser alterado também de maneira compensatória (Chiapparini et al, 2004).

Alguns fatores podem influenciar num risco maior de ocorrência de CPPD, e podem ser classificados em: relacionados ao paciente e relacionados à técnica de punção. No entanto, ainda que sabidamente esses fatores possam aumentar a chance da complicação, características fisiológicas possivelmente desconhecidas podem sugerir porque determinados pacientes apresentam CPPD, ainda que não façam parte de nenhum grupo de risco e a técnica de punção seja exemplar, e outros pacientes com vários fatores complicadores e técnica de punção difícil não apresentem a complicação (Clark et al., 1996).

Dentre os fatores relacionados ao paciente, pesquisas relacionaram a maior casuística de CPPD entre jovens adultos, diminuindo o risco com o avançar da idade. Isso pode ser devido à menor elasticidade da dura-máter, que dificulta a perda de LCS pelo orifício de perfuração, à menor reatividade dos vasos intracranianos à hipotensão/hipovolemia do LCS, e também devido à maior resistência extradural vertebral, que diminui a capacidade desse espaço em conter o LCS que estaria vazando. As crianças, por possuírem fisiologicamente menor pressão liquórica, e também baixa pressão hidrostática na região lombar, em comparação com um adulto, ao assumirem posição ereta, também apresentam baixa incidência de CPPD. O gênero feminino também é um fator de risco independente para CPPD, e pode ser explicado por diferenças fisiológicas e anatômicas, além de fatores psicossociais, em relação ao gênero masculino. Mulheres gestantes também possuem um risco maior, pois as alterações fisiológicas relacionadas à gestação, idade jovem, aumento da pressão liquórica no final da gestação e diminuição da sua densidade, e manobra de Valsalva (que decorre da expiração forçada contra a glote fechada, o que faz a pressão intraabdominal se elevar) durante o trabalho de parto podem estar associadas ao maior risco de extravasamento do LCS. Além da CPPD, mulheres gestantes também apresentam risco maior de complicações concomitantes, como hematoma subdural intracraniano, trombose venosa cerebral e apoplexia pituitária (hemorragia na hipófise). Outros fatores relacionados ao 
paciente que também podem aumentar o risco de CPPD são: história prévia de CPPD, baixo índice de massa corporal, obesidade mórbida e pacientes com cefaleia crônica (Lybecker et al., 1990; Choi et al., 2003; Ebinger et al, 2004; Wu et al., 2006).

Dentre os fatores relacionados à técnica de punção, o que mais se vincula à incidência de CPPD é o calibre da agulha. Quando mais calibrosa a agulha, mais tempo a trama tecidual da dura-máter leva para se reorganizar, portanto é maior o risco de extravasamento liquórico mais rápido do que sua produção, levando à CPPD. A solução para isso apenas parece simples, pois agulhas mais finas requerem precisão e habilidade maiores do profissional. O tipo do bisel também influencia na incidência de CPPD: o do tipo Quincke é fino, cortante e de fácil manuseio, no entanto, uma vez que corta o tecido da meninge, sua reestruturação é mais lenta e o risco de CPPD aumenta. O bisel do tipo "ponta de lápis", que está presente nas agulhas Whitacre e Sprotte, não é cortante, dessa forma divulsiona as fibras meningeais, favorecendo edema e reação inflamatória local, o que auxilia no fechamento do orifício de perfuração e causa menor perda liquórica. A direção com que o bisel das agulhas cortantes é inserido na dura-máter também é importante, pois se o corte for feito perpendicularmente à disposição das fibras das meninges, a reorganização tecidual é mais lenta; se o corte for paralelo à disposição das fibras, o risco de perda liquórica diminui. O modo de inserção da agulha também é algo que deve ser considerado importante, pois inserções paramedianas (em ângulo de $30^{\circ}$ ) perfuram a dura-máter e a aracnoide em ângulos diferentes, o que produz um mecanismo de válvula, que ajuda a impedir o fluxo do LCS pelo orifício de perfuração. As inserções medianas (em ângulo de $90^{\circ}$ ) possuem um risco maior de predisposição à CPPD (Turnbull \& Sheperd, 2003; Bezov et al., 2010).

Uma vez diagnosticada a CPPD, a conduta é determinada de acordo com o quadro doloroso apresentado e referido pelo paciente. A intervenção terapêutica ideal é a oclusão do orifício que está permitindo a perda liquórica, pois o tratamento apenas sintomático da cefaleia pode aumentar o desenvolvimento de complicações intracranianas, uma vez que a cefaleia ortostática serve como um sinal de alerta e proteção ao obrigar o paciente a permanecer em posição horizontal. A oclusão do orifício é rotineiramente realizada através de tampão sanguíneo peridural (blood patch), para que haja coagulação da abertura dural. A técnica inclui coleta de aproximadamente 20 mililitros de sangue autólogo e posterior injeção lenta deste sangue no espaço peridural, no mesmo nível da punção anterior (Safa-Tisseront et al., 2001; Boonmak \& Boonmak, 2010).

A Sociedade Internacional de Cefaleia definiu a CPPD como um quadro de evolução curta e auto resolutivo, surgindo até sete dias após o procedimento de punção dural e que costuma desaparecer em até duas semanas após a punção. No entanto, em alguns casos, a dor pode persistir por várias semanas ou meses (Barbosa, 2011). Dessa forma, é importante investigar a incidência de CPPD e levantar dados que podem auxiliar na avaliação dos motivos de ocorrência da complicação, melhoria das técnicas de punção lombar e maior apuração de fatores preditivos.

Assim, o objetivo do presente trabalho foi investigar a incidência e prevalência da Cefaleia Pós-punção Dural (CPPD) tratada com tamponamento sanguíneo peridural, associada a fatores clínicos e sociodemográficos em um hospital na cidade de Três Lagoas/MS.

\section{Metodologia}

Para obtenção dos dados necessários foi estabelecido parceria com um hospital da cidade de Três Lagoas-MS que consentiu em ceder seus dados ambulatoriais, além da submissão do projeto ao Comitê de Ética e Pesquisa (CEP). O protocolo submetido ao CEP concedeu parecer substanciado de aprovação ${ }^{\circ}$ 3.620.593. Ressaltamos que todos os dados obtidos foram totalmente respeitados quanto ao sigilo, uma vez que os nomes não foram relatados e tampouco houve contato, ou entrevista, com qualquer paciente. Além disso, estimamos os preceitos legais e éticos da resolução 466/12 pertencente ao Conselho Nacional de Saúde, sendo resguardada a confidencialidade dos dados fornecidos, utilizando-os somente para fins acadêmicos. 
Desta maneira, as etapas para a realização deste trabalho foram: 1- avaliação dos prontuários dos pacientes submetidos ao procedimento de tampão sanguíneo (blood patch) no período de 01/01/2011 a 03/04/2019; 2- análise das informações contidas nos prontuários que relacionavam ocorrência de cefaleia pós-punção, baseando-se na realização do procedimento de tampão sanguíneo e cirurgia prévia com anestesia subaracnóidea; 3- verificação dos eventos relacionados a cefaleia, procedimento de tampão sanguíneo e cirurgia prévia com fatores de riscos como características fisiológicas do paciente, gênero, idade, tipo de material utilizado na técnica de punção e qual foi a cirurgia previamente realizada.

Os dados foram analisados para que pudéssemos plotar gráficos, a prevalência e a incidência dos casos investigados. Os resultados das análises foram comparados aos dados da literatura nacional ou internacional dos últimos 20 anos. Foram excluídos os prontuários onde não havia informação sobre realização ou não de cirurgia prévia, tipo de cirurgia e procedimento anestésico, e material utilizado.

\section{Resultados e Discussão}

No período analisado, foram identificados 84 procedimentos de tamponamento sanguíneo peridural. No entanto, 27 destes pacientes foram excluídos das análises, pois 13 foram submetidos à cirurgia prévia com anestesia peridural, e não subaracnoidea, e os outros 14 tinham informações incompletas no prontuário, impossibilitando a inclusão no estudo. Dessa forma, no período de 01/01/2011 a 03/04/2019, chegamos a um total de 57 pacientes, pois além de submeteram-se ao procedimento de tamponamento sanguíneo peridural também realizaram à cirurgia prévia com anestesia subaracnóidea. Ao calcular a prevalência, chegamos a um valor de $0,25 \%$, uma vez que foram realizadas 22.655 raquianestesias no período analisado.

Em relação ao tipo de dor, não foi possível identificarmos padrões e relacioná-los aos descritos na literatura, uma vez que os prontuários não tinham essa informação. Esse problema repetiu-se em vários outros momentos (inclusive foi um dos critérios de exclusão de casos, quando dados de cirurgia prévia não foram descritos, por exemplo), e devemos ressaltar a importância do preenchimento adequado e o mais detalhado possível dos prontuários hospitalares, pois a falta de informações impede a correta alimentação de bancos de dados epidemiológicos.

O mesmo ocorreu com a informação sobre tempo de dor. Não havia descrito o tempo de queixa do paciente, bem como intensidade, que é um dado clínico importante, pois o tratamento da CPPD inclui alívio sintomático quando a dor não é importante. Dessa forma, utilizamos apenas os dados de tempo decorrido entre a intervenção cirúrgica prévia e a realização do tamponamento sanguíneo peridural (Figura 1). Admitindo esse tempo como período de dor crítica, pudemos notar que 52 pacientes apresentaram sintomas de CPPD em até 5 dias após a punção dural, necessitando da intervenção com tamponamento, o que é esperado de acordo com a literatura (Netto et al, 2010; Drake, 2015, Imbelloni, 2013).

Quanto à idade, 36 pacientes tinham idade entre 21 e 40 anos (Figura 2), corroborando os dados atuais que afirmam que a CPPD é mais comum em adultos jovens (Lybecker et al., 1990; Choi et al., 2003; Ebinger et al, 2004; Wu et al., 2006; Netto et. al, 2010). Em relação ao gênero, 49 pacientes eram mulheres, fato, inclusive, encontrado como fator de risco pra CPPD (Imbelloni, 2013). Um fato interessante é que 30 dessas pacientes fizeram cirurgia por motivo ginecológico/obstétrico, evento que pode colaborar para essa casuística maior de CPPD no sexo feminino (Figura 3). É bem descrito na literatura (Bardon et al., 2016) que mulheres gestantes possuem risco aumentado para desenvolvimento de CPPD após punção dural, e, em nossa pesquisa, 9 das 30 cirurgias ginecológicas/obstétricas eram cesáreas.

Sobre o material utilizado na anestesia subaracnoidea realizada para o procedimento cirúrgico (prévio ao tamponamento), em todos os pacientes foram utilizadas agulhas com bisel do tipo cortante (Quincke), o que pode prolongar o tempo de recuperação do tecido dural e aumentar os riscos de CPPD (Haller et al., 2018). Quanto ao calibre da agulha, todos os 
pacientes foram submetidos à punção com agulha $27 \mathrm{G}$, que é considerado um calibre fino, e apenas 2 pacientes precisaram de uma nova tentativa com agulha $25 \mathrm{G}$, que se trata de um calibre um pouco maior. Não foi possível analisar quantas tentativas foram realizadas até que a punção para a anestesia subaracnoidea ocorresse, pois esses dados também não estavam presentes nos prontuários.

Figura 1. Gráfico demonstrando o intervalo, em dias, entre a intervenção cirúrgica com anestesia subaracnóidea e o procedimento de tamponamento sanguíneo subdural. Acima de cada coluna, observa-se o número de pacientes que apresentou determinado intervalo de dias (no eixo $\mathrm{x}$ ) entre os procedimentos.

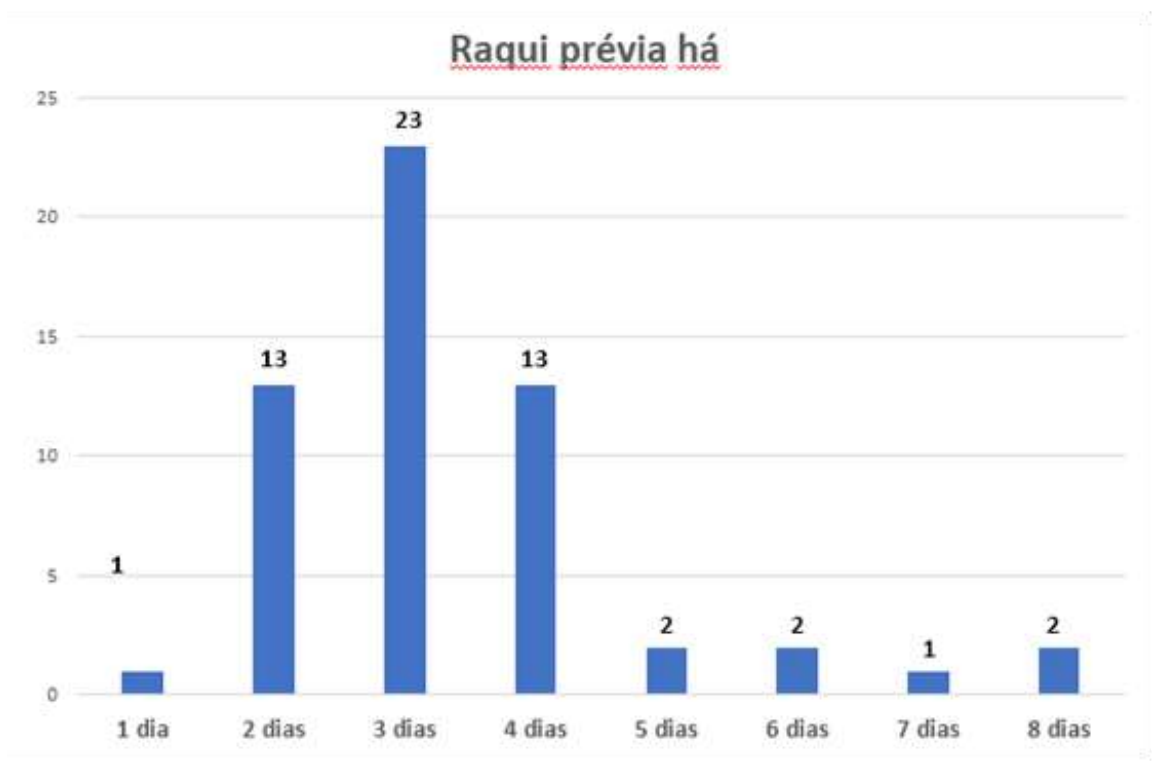

Fonte: Autores.

Figura 2: Gráfico demonstrando a quantidade de pacientes (números em negrito em frente às barras) pertencente a cada faixa de idade (eixo y) que foram submetidos ao tamponamento sanguíneo peridural após cirurgia realizada com anestesia subaracnoidea.

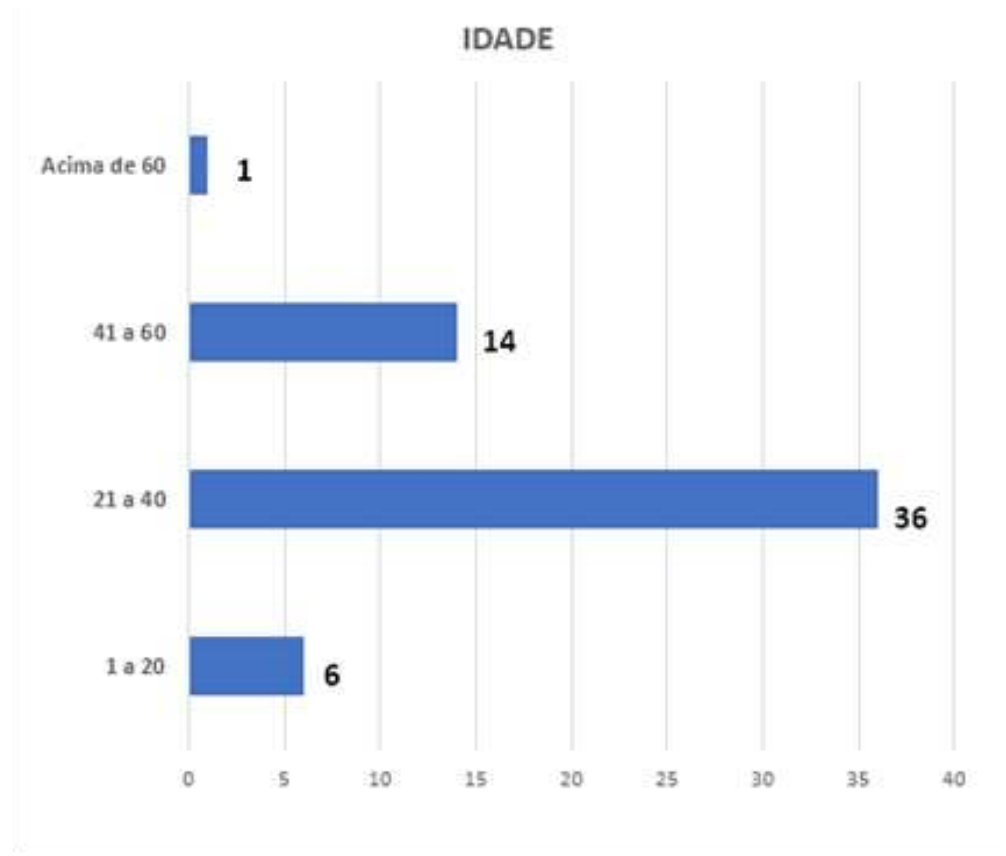

Fonte: Autores. 
Também não pudemos analisar a história prévia de CPPD, procedimentos realizados para alívio da dor além do tamponamento sanguíneo peridural, eficiência do tamponamento realizado e outros dados clínicos dos pacientes, pois esses dados estavam ausentes dos prontuários. Mais uma vez ressaltamos a importância do preenchimento adequado e completo da história clínica do paciente, isso é fundamental para a construção de um melhor atendimento, seguimento e compreensão de várias morbidades.

Figura 3: Gráfico demonstrando o número de pacientes (número em negrito) pertencente ao gênero masculino e ao gênero feminino que foram submetidos ao tamponamento sanguíneo peridural após cirurgia com anestesia subaracnoidea. Também está representada a porcentagem de cada categoria em relação ao número total de pacientes do estudo (57 pacientes).

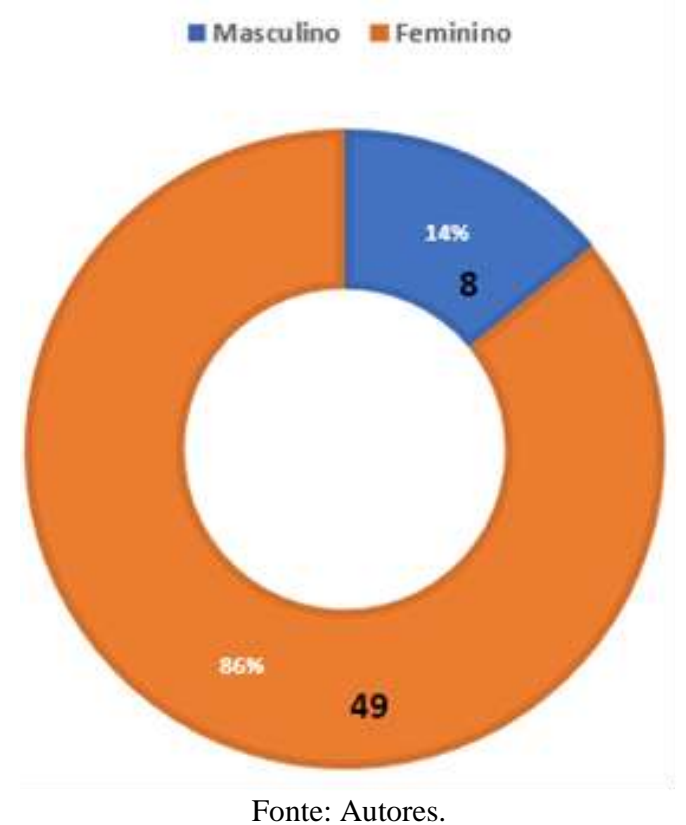

Sobre a incidência anual da CPPD no hospital referente ao estudo, observamos que foi oscilatória, e o número de casos por ano investigado é descrito na Tabela 1. Apesar de notarmos uma certa tendência ao aumento do número de tampões a cada ano, precisamos reforçar que os dados nos prontuários precisam ser melhor descritos, uma vez que até mesmo a literatura não encontra consenso ao descrever dados epidemiológicos de CPPD. Essa dificuldade também é sustentada pelo fato de que os hospitais podem ter protocolos próprios e diferentes quanto à conduta frente à CPPD, inclusive os critérios diagnósticos podem variar entre os centros hospitalares (Lavi et al., 2010; Hammond et al., 2011; Peralta et al., 2015).

Tabela 1: Incidência anual de tamponamentos sanguíneos peridurais, realizados após cirurgia com anestesia subaracnoidea, num hospital da cidade de Três Lagoas/MS.

\begin{tabular}{|l|l|}
\multicolumn{1}{|c|}{ Ano } & \multicolumn{1}{c}{ Número de tamponamentos realizados } \\
\hline 2011 & 0 \\
\hline 2012 & 1 \\
\hline 2013 & 5 \\
\hline 2014 & 1 \\
\hline 2015 & 6 \\
\hline 2016 & 11 \\
\hline 2017 & 18 \\
\hline 2018 & 9 \\
\hline 2019 (até $03 / 04)$ & 6 \\
\hline
\end{tabular}




\section{Conclusão}

A CPPD mostrou-se uma complicação frequente (de acordo com os dados epidemiológicos existentes na literatura em comparação aos dados encontrados neste trabalho) no hospital da cidade de Três Lagoas/MS referente a este estudo, e a realização de tamponamento sanguíneo peridural para tratamento de CPPD é comum, mas sem a garantia de resolução definitiva. A necessidade da adoção constante de medidas profiláticas, com evidências científicas, que possam reduzir a incidência da complicação é absoluta, uma vez que o retorno do paciente ao centro cirúrgico é acompanhado de riscos infectopatológicos, psicossociais e impactos socioeconômicos. Ademais, notou-se a extrema necessidade do preenchimento rico e adequado dos dados clínico do paciente em seu prontuário médico, uma vez que essa prática traz benefícios para a saúde do indivíduo, da comunidade, auxilia no desenvolvimento de manuais de conduta, alimenta dados epidemiológicos e é imprescindível na melhor compreensão de diversas patologias, como é o caso da CPPD.

\section{Referências}

Barbosa, F. T. (2011). Cefaleia após Anestesia Subaracnóidea com Sete Meses de Evolução: Relato de Caso. Revista Brasileira Anestesiologia, 61(3), 357359.

Bezov, D., Lipton, R. B. \& Ashina, S. (2010). Post-dural puncture headache: part I diagnosis, epidemiology, etiology, and pathophysiology. Headache: The Journal of Head and Face Pain, 50(7), 1144-1152.

Boonmak, P. \& Boonmak, S. (2010). Epidural blood patching for preventing and treating post-dural puncture headache. Cochrane Database of Systematic Reviews, 1.

Chiapparini, L., Ciceri, E., Nappini, S., Castellani, M. R., Mea, E., Bussone, G., \& Savoiardo, M. (2004). Headache and intracranial hypotension: neuroradiological findings. Neurological Sciences, 25(3), s138-s141.

Choi, P. T., Galinski, S. E., Takeuchi, L., Lucas, S., Tamayo, C. \& Jadad, A. R. (2003). PDPH is a common complication of neuraxial blockade in parturients: a meta-analysis of obstetrical studies. Canadian Journal of Anesthesia, 50(5), 460-469.

Clark, J. W., Solomon, G.D., Senanayake, P. D. \& Gallagher, C. (1996). Substance P concentration and history of headache in relation to postlumbar puncture headache: towards prevention. Journal of Neurology, Neurosurgery \& Psychiatry, 60(6), 681-683.

Drake, R. L., Vogl, A. W. \& Mitchell, A. W. M. (2015). Gray Anatomia para estudantes. Elsevier.

Ebinger, F., Kosel, C., Pietz, J. \& Rating, D. (2004). Headache and backache after lumbar puncture in children and adolescents: a prospective study. Pediatrics, 113(6), 1588-1592.

Falk, S. A., Fleisher, L. A. \& Chen, W. (2017). Overview of anesthesia. UpToDate. https://www. uptodate.com/contents/overview-of-anesthesia.

Ganem, E. M., Castiglia, Y. M. M. \& Vianna, P. T. G. (2002). Spinal anesthesia-induced neurological complications. Revista brasileira de anestesiologia, 52(4), 471-480, 2002.

Haller, G., Cornet, J., Boldi, M-O., Myers, C., Savoldelli, G. \& Kern, C. (2018). Risk factors for post-dural puncture headache following injury of the dural membrane: a root-cause analysis and nested case-control study. International journal of obstetric anesthesia, 36, 17-27.

Hammond, E. R., Wang, Z., Bhulani, N., McArthur, J. C. \& Levy, M. (2011). Needle type and the risk of post-lumbar puncture headache in the outpatient neurology clinic. Journal of the neurological sciences, 306(1-2), 24-28, 2011.

Imbelloni, Luís Eduardo. (2013) Raquianestesia. Elsevier.

Ituk, U., MBBS, FCARCSI \& Wong, C. A. (2018). Overview of neuraxial anesthesia. UpToDate. https://www. uptodate.com/contents/overview-of-neuraxialanesthesia.

Lavi, R., Rowe, J. M. \& Avivi, I. (2010). Lumbar puncture: it is time to change the needle. European Neurology, 64(2), 108-113.

Lybecker, H., Møller, J. T., May, O. \& Nielsen, H. K. (1990). Incidence and prediction of postdural puncture headache. A prospective study of 1021 spinal anesthesias. Anesthesia and analgesia, 70(4), 389-394.

Netto, B. I., Catharino, A. M. da. S., Crasto, M. do C. V. de, Pires, M. L. E., Silva, M. G., Salles, L. C. B. \& Melo, C. (2010). Cefaleia Pós-Raquianestesia: fatores de risco associados e prevenção de sua ocorrência-Atualização. Rev. Neurociência, 18, 406-410.

Peralta, F., Higgins, N., Lange, E., Wong, C. A. \& McCarthty, R. J. (2015). The relationship of body mass index with the incidence of postdural puncture headache in parturients. Anesthesia \& Analgesia, 121(2), 451-456.

Safa-Tisseront, V., Thormann, F., Malassiné, P., Henry, M., Riou, B., Coriat, P. \& Seebacher, J. (2001). Effectiveness of epidural blood patch in the management of post-dural puncture headache. Anesthesiology: The Journal of the American Society of Anesthesiologists, 95(2), 334-339. 
Research, Society and Development, v. 11, n. 1, e16211124576, 2022 (CC BY 4.0) | ISSN 2525-3409 | DOI: http://dx.doi.org/10.33448/rsd-v11i1.24576

Turnbull, D. K. \& Shepherd, D. B. (2003). Post-dural puncture headache: pathogenesis, prevention and treatment. British journal of anaesthesia, 91(5), 718729.

Wu, C. L., Rowlingson, A. J., Cohen, S. R., Michaels, R. K., Courpas, G. E., Joe, E. M. \& Liu, S. S. (2006). Gender and post-dural puncture headache. Anesthesiology: The Journal of the American Society of Anesthesiologists, 105(3), 613-618,

Wu, C. L., Christo, P., Richman, J. M. \& Hsu, W. (2004). Postdural puncture headaches: an overview. The International Journal of Pain Medicine And Paliative Care, 3(2), 53-7. 